\title{
Activation of Tryptophan and Phenylalanine Catabolism in the Remission Phase of Allergic Contact Dermatitis: A Pilot Study
}

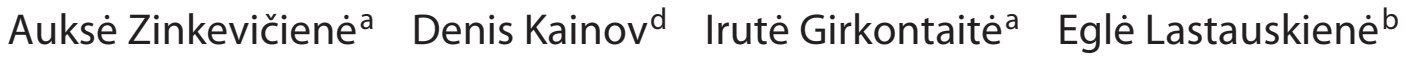 \\ Violeta Kvedarienèc $^{c}$ Yu Fu $^{d}$ Simon Anders ${ }^{d}$ Vidya Velagapudi ${ }^{d}$ \\ ${ }^{a}$ Department of Immunology, State Research Institute Centre for Innovative Medicine, ${ }^{b}$ Department of \\ Microbiology and Biotechnology, Faculty of Natural Sciences, and ${ }^{\mathrm{C}}$ Faculty of Medicine, Vilnius University, \\ Vilnius, Lithuania; ${ }^{d}$ Institute for Molecular Medicine Finland, FIMM, University of Helsinki, Helsinki, Finland
}

\section{Key Words}

Allergic contact dermatitis · Metabolites · Tryptophan .

Phenylalanine

\begin{abstract}
Background: Allergic contact dermatitis (ACD) is an inflammatory skin disease caused by repeated skin exposure to contact allergens. The severity and duration of this disease are associated with many different factors. Some of these factors may represent markers for monitoring disease activity and the individual response to an intervention. Methods: We used a targeted metabolomics approach to find such factors in the serum of individuals with ACD. Metabolomics profiles were examined and compared in the acute phase of the disease and also in the absence of disease activity. Results: Our study identified a significant remission phase of ACDassociated systemic biochemical shifts in 2 metabolic pathways: tryptophan-kynurenine and phenylalanine-tyrosine. Conclusions: Although the responsible mechanisms are unclear, these results suggest that the remission phase of ACD is linked to tryptophan metabolism via kynurenine and phenylalanine-tyrosine pathways. However, further replication studies with a larger number of subjects and their subgroups are necessary to validate our results. These studies may pro-
\end{abstract}

vide a new perspective with which to understand the mechanism of and find potential biomarkers of ACD, as well as a new reference for personalized treatment.

(c) 2016 S. Karger AG, Base

\section{Introduction}

Allergic contact dermatitis (ACD) is one of the most common inflammatory skin diseases induced by exposure to an environmental agent. It is an important occupational disease affecting the general population since general skin sensitizers are metals, fragrances, hair dye ingredients, and preservatives. In Europe $20-25 \%$ of the population is sensitive to at least 1 contact allergen. The immunologic mechanisms by which contact hypersensitivity occur are complex, involve Th1 and Th2 immune responses and are the result of numerous immune cells, cytokines, and chemokines. Numerous types of regulatory cells control the severity and duration of these reactions and the only available etiologic treatment of ACD is elimination of the skin sensitizer $[1,2]$. Complex interactions among genetic, epigenetic and environmental factors influence ACD. For a better understanding of the pathogenesis and treatment of this disease, new systemic

\section{KARGER}

E-Mail karger@karger.com

www.karger.com/iaa
(C) 2016 S. Karger AG, Basel

$1018-2438 / 16 / 1704-0262 \$ 39.50 / 0$
Correspondence to: Dr. Auksė Zinkevičienė

Department of Immunology, State Research Institute Centre for Innovative Medicine Santariškiu str. 5

LT-08406 Vilnius (Lithuania)

E-Mail aukse.zinkeviciene@ gmail.com 
Fig. 1. ACD cases and controls and their susceptibility to sensitization with different allergens. Patch test reactions were graded from no reaction $(0)$ to $3+$.

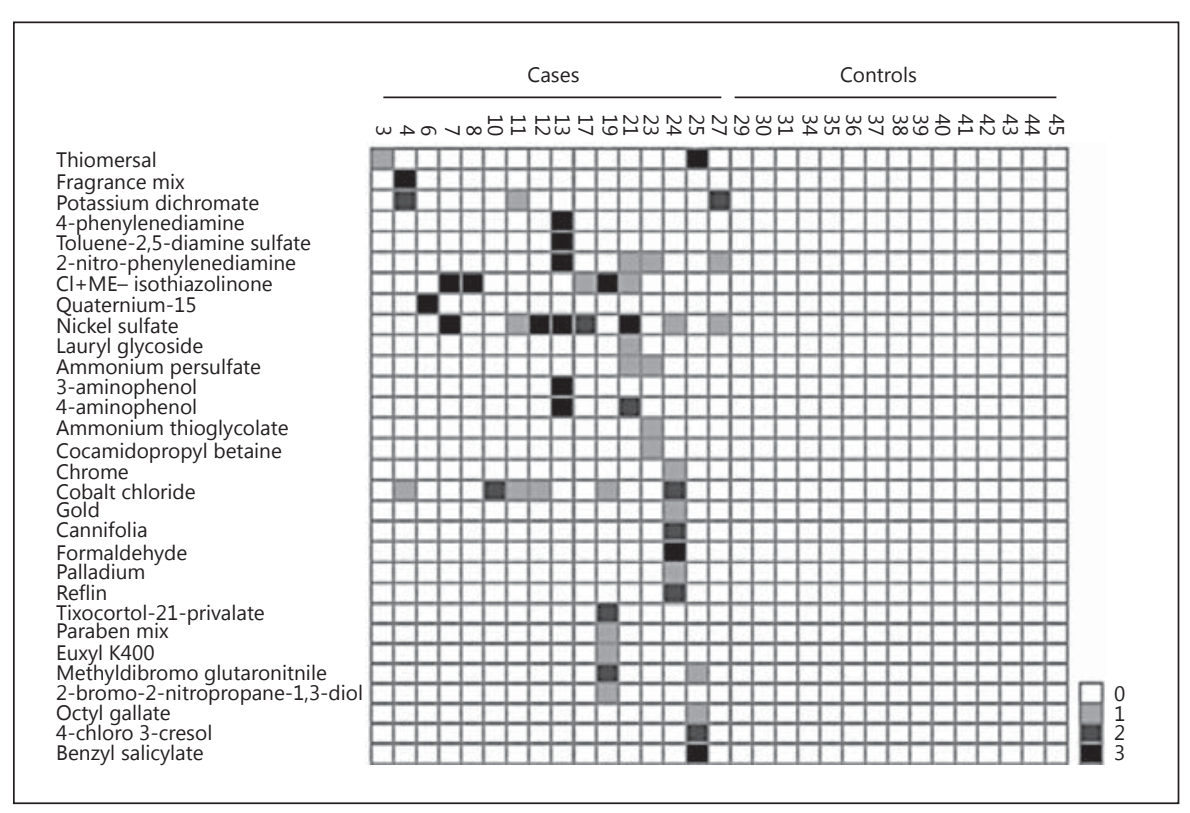

approaches are required. The identification of biochemical biomarkers enable the the risk of developing the disease to be determined, along with a prediction of the disease progression, monitoring of the disease activity, and clinical response to an intervention.

In order to identify novel biomarkers in biological samples, researchers have begun to employ 'omics' approaches in combination with biostatistical methods. Metabolomics is the systematic study of the global profiles of all metabolites, i.e. small molecules that are involved in the metabolism and are the end points of cellular regulation $[3,4]$. Systematic metabolomics analysis at the right time points can provide new molecular insights into the elucidation of metabolic regulations underlying the disease development and improve risk prediction, which is crucial for the advancement of prevention and treatment strategies. Changes in the metabolite concentration may also reflect disease progression or predict responses to drugs. This approach is becoming increasingly popular in clinical studies with the suggestion that metabolomics could be a powerful tool for discovering new biomarkers, the characterization of different clinical phenotypes, early diagnosis or developing strategies for disease treatment. Metabolomics has been used in some allergic diseases, such as atopic dermatitis, to investigate the relevance of this method as a novel diagnostic tool [5].

The purpose of this pilot study was to identify differential blood metabolomics profiles in patients with ACD compared with healthy individuals. In this study, we employed a targeted tandem mass spectrometry metabolomics approach to analyze the systemic biochemical shifts associated with the course of ACD. Metabolomics profiles were also examined and compared in the acute phase of the disease and in the absence of disease activity in order to provide a new perspective with which to understand the mechanism associated with disease development.

\section{Materials and Methods}

\section{Study Group}

Sixteen adults ( 15 females and 1 male, aged $21-54$ years, mean age $33.1 \pm 10.6$ years) were consulted for ACD at the Centre of Diagnosis and Treatment of Allergic Diseases, Vilnius, Lithuania. During the first visit (acute phase of ACD) the blood samples were taken and treatment was prescribed to the patients. During the second visit (remission phase of ACD), blood samples were collected again from the same individuals. Patch tests were performed in both groups - the case and control groups - using European standards (S-1000; Chemotechnique Diagnostics, Vellinge, Sweden) and suspected allergens in nonirritant concentrations made ex tempore. Tests for other allergens were performed according to clinical needs. Allergens were applied to the upper back of participants using the Finn Chambers (SmartPractice, Phoenix, Ariz., USA) system for 2 days. Readings according to international criteria were performed on days 2, 3, and 7 [6]. The patch test reactions were graded by severity from no reaction (0) to $3+$. Importantly, patients did not use local and/or systemic corticosteroids for at least 1 month, and treatment with antihistamines was stopped for 4-6 days before patch testing. The control group also consisted of 


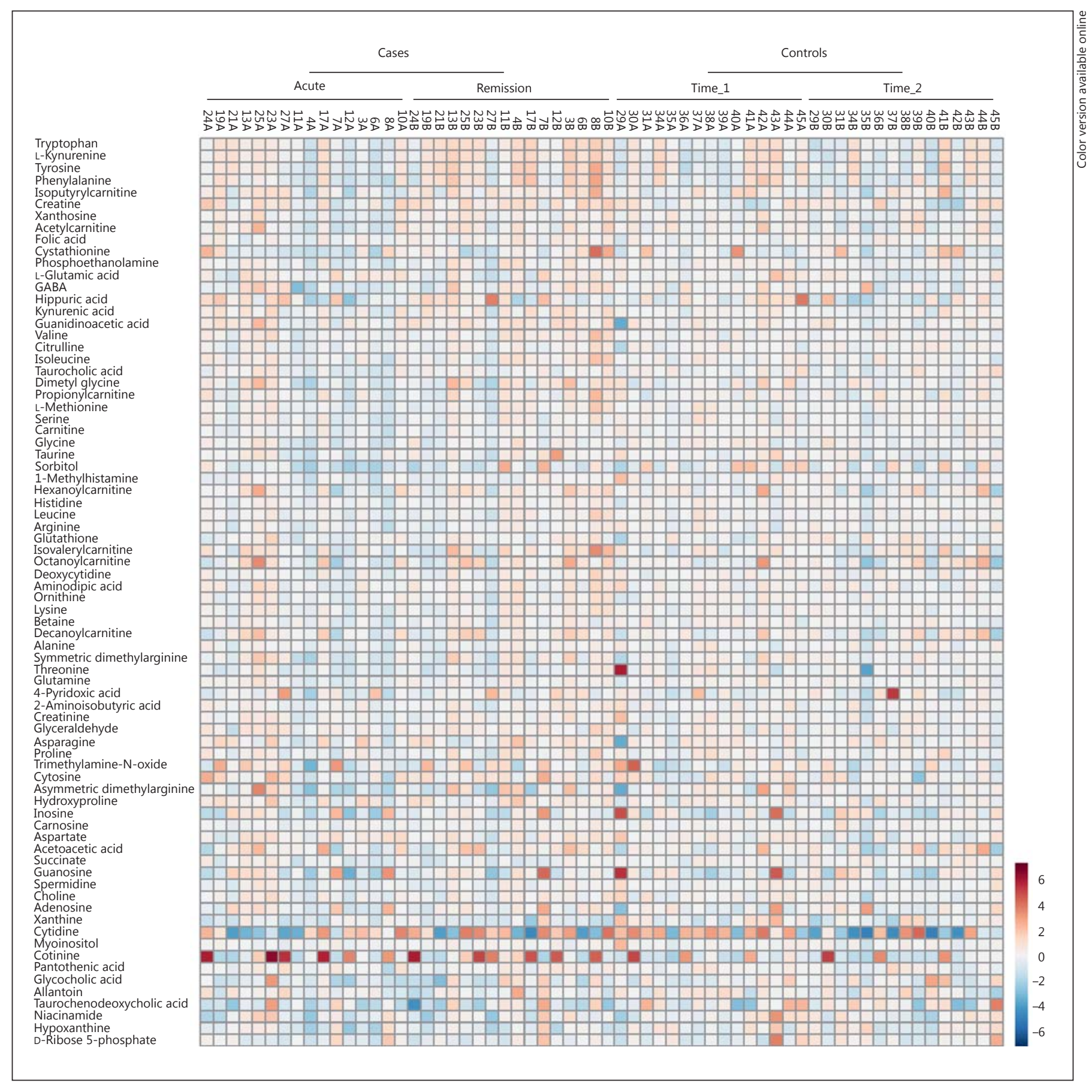

Fig. 2. Heat map of 76 metabolites in $16 \mathrm{ACD}$ patients and 15 controls.

15 healthy volunteers ( 14 females and 1 male; aged $21-35$ years, mean age $26.4 \pm 7.8$ years) without a previous history of allergic disorders. We also collected blood samples twice from these healthy volunteers, with a 1-month interval between sampling. The study was approved by the Lithuanian Bioethics Committee. Written consent was obtained from each participant.

\section{Metabolomics Analyses}

Briefly, $10 \mu \mathrm{l}$ of labeled internal standard mixture was added to $100 \mu \mathrm{l}$ of serum sample. Metabolites were extracted by adding 4 parts of the $100 \%$ acetonitrile $+1 \%$ formic acid solvent (1:4, sample:extraction solvent). The collected extracts were dispensed on an Ostro ${ }^{\mathrm{TM}}$ 96-well plate (Waters Corporation, Milford, Conn., 
Table 1. Significantly enriched metabolites in the cobalt chloride subgroup (LIMMA test; $\mathrm{p}<0.1$ )

\begin{tabular}{llcllll}
\hline Metabolite & Acute phase & Remission phase & Average expression & Fold & $\mathrm{p}$ & Adjusted $\mathrm{p}$ \\
\hline Hydroxyproline & -0.06962153 & 1.0314158 & 4.728607 & 9.584622 & 0.0003515144 & 0.02706661 \\
Hypoxanthine & -1.17669988 & -0.9929157 & 6.369091 & 7.128930 & 0.0020837468 & 0.08022425 \\
\hline
\end{tabular}

The acute $(n=6)$, remission $(n=6)$ and control $(n=30)$ groups were compared.

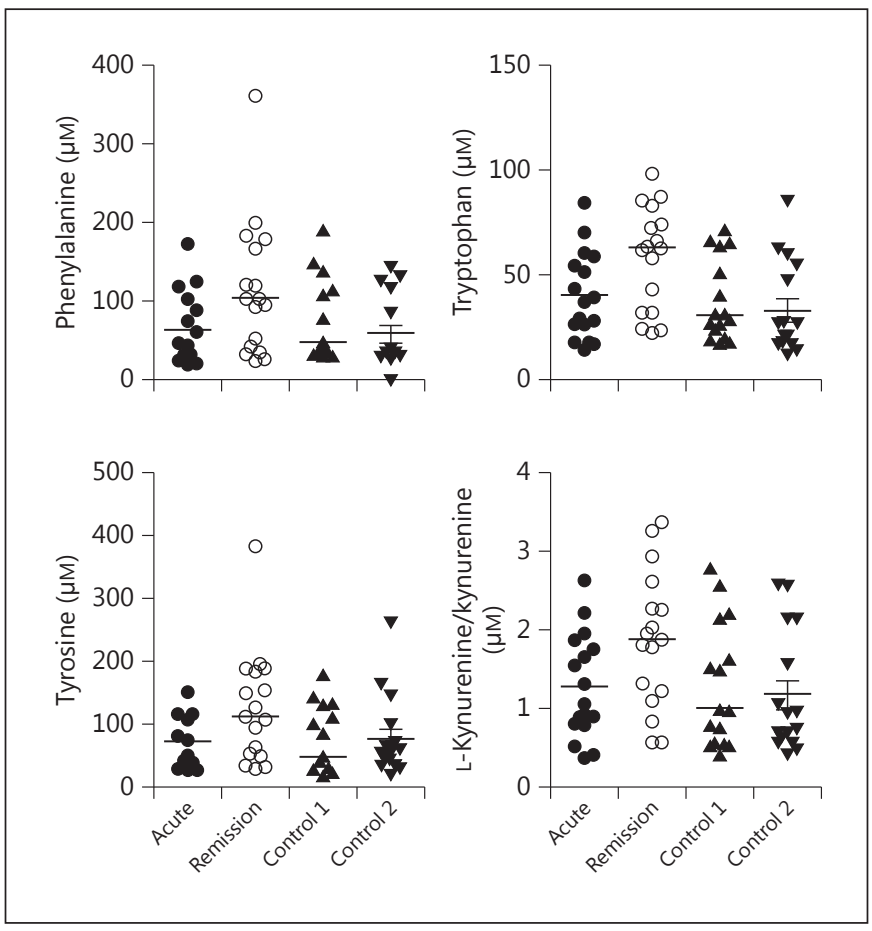

Fig. 3. Serum levels of tryptophan, L-kynurenine, tyrosine and phenylalanine in patients with ACD and healthy controls.

USA) and filtered by applying a vacuum at a delta pressure of 300$400 \mathrm{mbar}$ for $2.5 \mathrm{~min}$. A detailed protocol about the analytical conditions, instrument parameters, and the list of measured metabolites are given elsewhere [7].

\section{Results}

We recruited 16 patients with a diagnosis of ACD. Patch tests confirmed the preliminary diagnosis and showed positive reactions to 1 or more sensitizers. There were a total of $62 \mathrm{ACD}$ reactions, with the most frequent being nickel sulfate and cobalt chloride (fig. 1).

Tryptophan and Phenylalanine

Catabolism in ACD
Serum samples were collected from ACD patients during acute and remission phases of the disease, as well as from healthy volunteers with a 1-month interval. In total, 76 metabolites were profiled and we compared the concentrations of these metabolites in the serum samples of patients and controls (fig. 2). The levels of tryptophan, Lkynurenine, tyrosine and phenylalanine were significantly altered in ACD patients in comparison with healthy volunteers and differences in the same metabolite levels were seen in serum samples collected during the acute phase of ACD and during remission (fig. 3).

Analysis of metabolite levels in the most representative patient subgroups revealed that the patients with cobalt chloride allergy had significantly increased hydroxyproline and hypoxanthine levels in their serum (table 1). There were no other differences between initial results and results obtained after this subgrouping. Analysis of metabolite levels in the most representative patient subgroups did not yield any significant association with ACD.

\section{Discussion}

Metabolic profiling can be a powerful tool with which to gain insight into disease processes. Identification of metabolites displaying altered levels and their metabolic pathways can help the discovery of the new biomarkers, early diagnosis or developing strategies for disease treatment. The aim of this pilot study was with a targeted metabolomics approach to investigate the differences in the serum metabolome of ACD patients and to analyze the systemic biochemical shifts. We evaluated levels of metabolites in the sera of patients suffering from ACD, as well as in healthy controls. The results showed that levels of tryptophan, L-kynurenine and phenylalanine were significantly elevated in the remission phase of the patients as compared to healthy controls. We also compared metabolite levels in the serum of ACD patients during the 
Fig. 4. Metabolite levels that were elevated in patients during the remission phase of ACD.

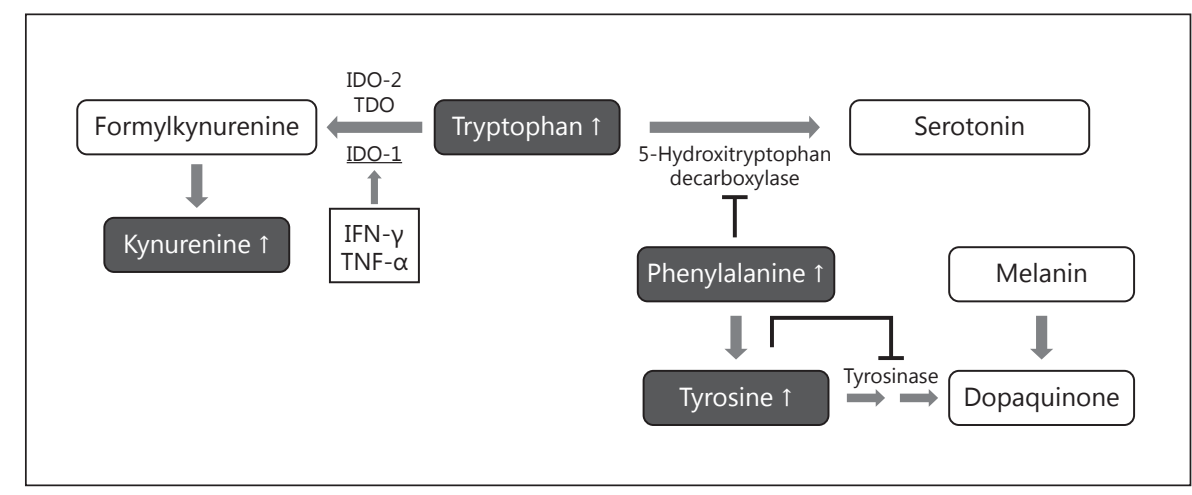

acute and remission phases of the disease. In our study, the remission phase of ACD phase was associated with an increase in 4 metabolites - tryptophan, L-kynurenine, phenylalanine and tyrosine - as compared to the acute phase of the disease.

Tryptophan and phenylalanine are essential amino acids that the human body is incapable of synthesizing. The increased serum levels of these amino acids could be related to an altered catabolism (fig. 4).

Tryptophan can be metabolized through different pathways in the body, but the kynurenine pathway is a major route for the nonprotein metabolism of this compound [8]. Shifts in tryptophan metabolism through the kynurenine pathway have been previously reported in cancer, asthma, dengue infection, Alzheimer disease, AIDS, Huntington disease, schizophrenia and depression [9-14]. There are 3 known enzymes that oxidize tryptophan in the kynurenine pathway: tryptophan 2,3-dioxygenase (TDO), the predominant enzyme indoleamine 2,3 -dioxygenase 1 (IDO-1) or IDO-2 [15, 16]. IDO is expressed in certain cells and tissues, particularly in antigen-presenting cells of lymphoid organs. There is a hypothesis that the expression of IDO in antigen-presenting cells may control autoreactive T cells [17]. Enzyme production markedly increases during infection or inflammation. In response to inflammatory stimuli, IDO functions as an immune regulator to maintain proinflammatory signaling. The hyperactivation of the IDO can be provoked by cytokines, chemokines and inflammation mediators [18]. The most potent inducer of IDO-1 in the kynurenine pathway is interferon gamma (IFN- $\gamma$ ). The other cytokine, tumor necrosis factor (TNF), stimulates and drastically potentiates IFN- $\gamma$-induced IDO- 1 activity [19]. ACD is a T cell-mediated inflammatory skin disease in the pathogenesis involving high levels of IFN- $\gamma$ and TNF- $\alpha$ [2]. During an immune response activated T cells produce IFN- $\gamma$, which induces IDO gene expression and leads to an increased tryptophan conversion to kynurenines. This tryptophan metabolic pathway is activated by proinflammatory stimuli, but metabolites deriving from the reactions of the kynurenine pathway induce lymphocyte apoptosis [20]. It has been discovered that kynurenine can enhance polarization of CD4 T cells to a Treg phenotype versus Th17 cells $[21,22]$. Stimulation of the kynurenine pathway was also shown to create an immune-suppressive milieu in tumor cells through secretion of immunosuppressive tryptophan metabolites that lead to the induction of $\mathrm{T}$ cell apoptosis and increased proliferation of immunosuppressive regulatory $\mathrm{T}$ cells [23]. Based on these findings, IDO1 is becoming established as a target for drug discovery in cancer [24]. In our study, serum levels of these metabolites were significantly increased during the remission ACD phase in comparison with controls and the acute phase samples. Based on these results, we hypothesize that in ACD the role of the kynurenine pathway consists in the ability of preventing tissue damage due to cytotoxic immune actions. Increased serum levels of these metabolites in the remission phase may reflect that clinically observed remissions of ACD do not match the immunological remission of this disease.

The other tryptophan metabolic route is the so-called methoxyindole pathway leading to serotonin formation. Serotonin is a neurotransmitter that has been associated with a wide variety of behaviors and many disorders, such as reward processing, sleep cycles, circadian rhythms, etc. $[25,26]$. There is evidence that in the skin serotonin exerts proinflammatory actions, modulates the immune response and contributes to chronic atopic dermatitis by inhibiting the apoptosis of immune cells $[27,28]$. The activation of the kynurenine pathway enzymes (TDO and IDO) could reduce the serotonin bio- 
synthesis. Additionally, in our study, during the ACD remission phase serum levels of phenylalanine were significantly increased in comparison with controls and the acute phase samples. There is evidence that high phenylalanine levels lead to disturbances in tryptophan metabolism since the 5-hydroxytryptophan decarboxylase (the enzyme involved in serotonin synthesis) activity is inhibited by the elevated blood levels of phenylalanine, so that little or no serotonin is formed [29]. All these findings suggest that the elevated serum tryptophan levels observed in our study may reflect the impairment of tryptophan conversion into serotonin and that tryptophankynurenine pathway activation may provide immunoprotective action in ACD.

In this study, the remission phase of ACD was associated not only with an increase in tryptophan, kynurenine and phenylalanine, but also with a significant increase in tyrosine serum levels. Tyrosine is a nonessential amino acid that is synthesized from phenylalanine by phenylalanine hydroxylase [30]. Tyrosine is also the precursor to melanin which is produced in specialized cell melanocytes [31]. It has been observed that inflammatory dermatoses are associated with changes in melanocyte function and hyperpigmentation is clinically observed in response to inflammation [32]. The first step of the biosynthetic pathway for melanin is catalyzed by tyrosinase. Tyrosine stimulates tyrosinase activity in a dose-dependent manner. At higher tyrosine concentrations, tyrosinase activity decreases and inhibits melanocyte proliferation [33]. In our study the observed increased serum tyrosine level during the remission phase of ACD led us to suggest that this increase could be related to decreased tyrosinase activity to avoid postinflammatory hyperpigmentation of the skin.

There are multiple pathways through which antigenpresenting cells modulate extracellular concentrations of amino acids to regulate $\mathrm{T}$ cell responses. A metabolic profiling approach provides a new perspective with which to understand the mechanism of ACD. The aim of this pilot study was to investigate metabolic variations in ACD patients with a targeted metabolomics approach. Metabolic differences were observed between ACD patients and healthy controls, and between remission and acute phases of the disease. These variations involved significant perturbations in tryptophan and phenylalanine metabolism. Although the responsible mechanisms are unclear, these results suggest that the remission phase of ACD is somehow linked to tryptophan metabolism via kynurenine and phenylalanine-tyrosine pathways. We suggest that observed metabolomic shifts reflect the disease progress or therapeutic response to treatment, and that levels of circulating metabolites could be useful for monitoring of the disease. Additional studies with larger cohorts will be needed to understand the significance of the observed amino acid shifts.

\section{Disclosure Statement}

All authors state that they have no conflicts of interest to declare. This study was supported by J. and A. Erkko foundation.

\section{References}

1 Thyssen JP, Linneberg A, Menne T, Johansen JD: The epidemiology of contact allergy in the general population - prevalence and main findings. Contact Dermatitis 2007;57:287299.

2 Vocanson M, Hennino A, Rozieres A, Poyet G, Nicolas JF: Effector and regulatory mechanisms in allergic contact dermatitis. Allergy 2009;64:1699-1714.

3 Sharon D, Chen R, Snyder M: Systems biology approaches to disease marker discovery. Dis Markers 2010;28:209-224.

4 Li S, Todor A, Luo R: Blood transcriptomics and metabolomics for personalized medicine. Comput Struct Biotechnol J 2016;14:1-7.

5 Huang Y, Chen G, Liu X, Shao Y, Gao P, Xin C, Cui Z, Zhao X, Xu G: Serum metabolomics study and eicosanoid analysis of childhood atopic dermatitis based on liquid chromatography-mass spectrometry. J Proteome Res 2014;13:5715-5723.
6 White JM: Patch testing: what allergists should know. Clin Exp Allergy 2012;42:180185.

7 Nikkanen J, Forsstrom S, Euro L, Paetau I, Kohnz RA, Wang L, Chilov D, Viinamaki J, Roivainen A, Marjamaki P, Liljenback $\mathrm{H}$, Ahola S, Buzkova J, Terzioglu M, Khan NA, Pirnes-Karhu S, Paetau A, Lonnqvist T, Sajantila A, Isohanni P, Tyynismaa $\mathrm{H}$, Nomura DK, Battersby BJ, Velagapudi V, Carroll CJ, Suomalainen A: Mitochondrial DNA replication defects disturb cellular dNTP pools and remodel one-carbon metabolism. Cell Metab 2016;23:635-648.

8 Ruddick JP, Evans AK, Nutt DJ, Lightman SL, Rook GA, Lowry CA: Tryptophan metabolism in the central nervous system: medical implications. Expert Rev Mol Med 2006;8:127.
9 Miyamoto S, Taylor SL, Barupal DK, Taguchi A, Wohlgemuth G, Wikoff WR, Yoneda KY, Gandara DR, Hanash SM, Kim K, Fiehn O: Systemic metabolomic changes in blood samples of lung cancer patients identified by gas chromatography time-of-flight mass spectrometry. Metabolites 2015;5:192-210.

10 Cui L, Lee YH, Kumar Y, Xu F, Lu K, Ooi EE, Tannenbaum SR, Ong CN: Serum metabolome and lipidome changes in adult patients with primary dengue infection. PLoS Negl Trop Dis 2013;7:e2373.

11 Oxenkrug GF: Genetic and hormonal regulation of tryptophan kynurenine metabolism: implications for vascular cognitive impairment, major depressive disorder, and aging. Ann NY Acad Sci 2007;1122:35-49.

12 Guillemin GJ, Brew BJ: Implications of the kynurenine pathway and quinolinic acid in Alzheimer's disease. Redox Rep 2002;7:199_ 206 
13 Guillemin GJ, Kerr SJ, Brew BJ: Involvement of quinolinic acid in aids dementia complex. Neurotox Res 2005;7:103-123.

14 Stoy N, Mackay GM, Forrest CM, Christofides J, Egerton M, Stone TW, Darlington LG: Tryptophan metabolism and oxidative stress in patients with Huntington's disease. J Neurochem 2005;93:611-623.

15 Takikawa O: Biochemical and medical aspects of the indoleamine 2,3-dioxygenase-initiated L-tryptophan metabolism. Biochem Biophys Res Commun 2005;338:12-19.

16 Metz R, Duhadaway JB, Kamasani U, LauryKleintop L, Muller AJ, Prendergast GC: Novel tryptophan catabolic enzyme IDO2 is the preferred biochemical target of the antitumor indoleamine 2,3-dioxygenase inhibitory compound D-1-methyl-tryptophan. Cancer Res 2007;67:7082-7087.

17 Munn DH, Shafizadeh E, Attwood JT, Bondarev I, Pashine A, Mellor AL: Inhibition of T cell proliferation by macrophage tryptophan catabolism. J Exp Med 1999;189:1363-1372.

18 Bianchi M, Bertini R, Ghezzi P: Induction of indoleamine dioxygenase by interferon in mice: a study with different recombinant interferons and various cytokines. Biochem Biophys Res Commun 1988;152:237-242.

19 Robinson CM, Hale PT, Carlin JM: The role of IFN- $\gamma$ and TNF- $\alpha$-responsive regulatory elements in the synergistic induction of indoleamine dioxygenase. J Interferon Cytokine Res 2005;25:20-30.

20 Ramanathan A, Wang C, Schreiber SL: Perturbational profiling of a cell-line model of tumorigenesis by using metabolic measurements. Proc Natl Acad Sci USA 2005;102: 5992-5997.
21 Opitz CA, Litzenburger UM, Sahm F, Ott M, Tritschler I, Trump S, Schumacher T, Jestaedt L, Schrenk D, Weller M, Jugold M, Guillemin GJ, Miller CL, Lutz C, Radlwimmer B, Lehmann I, von Deimling A, Wick W, Platten M: An endogenous tumour-promoting ligand of the human aryl hydrocarbon receptor. Nature 2011;478:197-203.

22 Mezrich JD, Fechner JH, Zhang X, Johnson BP, Burlingham WJ, Bradfield CA: An interaction between kynurenine and the aryl hydrocarbon receptor can generate regulatory $\mathrm{T}$ cells. J Immunol 2010;185:3190-3198.

23 Thaker AI, Rao MS, Bishnupuri KS, Kerr TA, Foster L, Marinshaw JM, Newberry RD, Stenson WF, Ciorba MA: IDO1 metabolites activate $\beta$-catenin signaling to promote cancer cell proliferation and colon tumorigenesis in mice. Gastroenterology 2013;145:416-425. e1-4.

24 Lob S, Konigsrainer A, Schafer R, Rammensee HG, Opelz G, Terness P: Levo- but not dextro-1-methyl tryptophan abrogates the IDO activity of human dendritic cells. Blood 2008; 111:2152-2154

25 Carley DW, Radulovacki M: Mirtazapine, a mixed-profile serotonin agonist/antagonist, suppresses sleep apnea in the rat. Am J Respir Crit Care Med 1999;160:1824-1829.

26 Kim TD, Kim JS, Kim JH, Myung J, Chae HD, Woo KC, Jang SK, Koh DS, Kim KT: Rhythmic serotonin $\mathrm{n}$-acetyltransferase mRNA degradation is essential for the maintenance of its circadian oscillation. Mol Cell Biol 2005; 25:3232-3246.
27 Soga F, Katoh N, Inoue T, Kishimoto S: Serotonin activates human monocytes and prevents apoptosis. J Invest Dermatol 2007;127: 1947-1955.

28 Ghia JE, Li N, Wang H, Collins M, Deng Y, El-Sharkawy RT, Cote F, Mallet J, Khan WI: Serotonin has a key role in pathogenesis of experimental colitis. Gastroenterology 2009; 137:1649-1660.

29 Loo YH: Serotonin deficiency in experimental hyperphenylalaninemia. J Neurochem 1974; 23:139-147.

30 Martinez A, Knappskog PM, Olafsdottir S, Doskeland AP, Eiken HG, Svebak RM, Bozzini M, Apold J, Flatmark T: Expression of recombinant human phenylalanine hydroxylase as fusion protein in Escherichia coli circumvents proteolytic degradation by host cell proteases: isolation and characterization of the wild-type enzyme. Biochem J 1995;306(pt 2):589-597.

31 Hirobe T: Structure and function of melanocytes: microscopic morphology and cell biology of mouse melanocytes in the epidermis and hair follicle. Histol Histopathol 1995;10: 223-237.

32 Hiramoto K, Kobayashi H, Ishii M, Sato E, Inoue $\mathrm{M}$ : Increased $\alpha$-melanocyte-stimulating hormone ( $\alpha-\mathrm{MSH})$ levels and melanocortin receptors expression associated with pigmentation in an NC/Nga mouse model of atopic dermatitis. Exp Dermatol 2010;19:132-136.

33 Slominski A, Costantino R: Molecular mechanism of tyrosinase regulation by L-DOPA in hamster melanoma cells. Life Sci 1991;48: 2075-2079. 